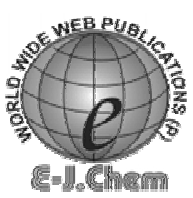

http://www.e-journals.net
ISSN: 0973-4945; CODEN ECJHAO

E-Journal of Chemistry

Vol. 5, No.2, pp.347-354, April 2008

\title{
Syntheses of 1, 2, 4 Triazole Derivatives and their Biological Activity
}

\author{
FREDDY H. HAVALDAR* and ABHAY R. PATIL \\ Nadkarny-Sacasa Research Laboratory, \\ Department of Chemistry, St. Xavier's College, Mumbai - 400001 \\ drfreddy_11@yahoo.co.in
}

Received 14 October 2007; Accepted 10 December 2007

\begin{abstract}
Cyclisation of [4-(4-oxo-2-phenyl-4H-quinazolin-3-yl)-phenoxy]-acetic acid- $N^{\prime}$-(substituted phenyl)-thiosemicarbazides with sodium metal in $99.0 \%$ ethanol gave 3-[4-(4-substituted phenyl-5-thioxo-4,5-dihydro- $1 H$-1,2,4 triazol-3-ylmethoxy)-phenyl]-2-phenyl-3H-quinazolin-4-one. The structures of the newly synthesized compounds have confirmed by IR, ${ }^{1} H$ NMR and Mass spectra. The compounds have also been screened for their biological activity.
\end{abstract}

Keywords: 2-Phenyl-4-(3H)-quinazolin-4-one, 1,2,4- Triazole derivatives, IR, Spectral studies, Biological activity.

\section{Introduction}

The chemistry of heterocycles is an interesting branch in organic chemistry. Quinazolinone derivatives derived from 2-phenyl-4H-3,1-benzoxazin-4-one are important compounds in chemistry and pharmacology. They have drawn much attention due to their broad range of pharmacological properties ${ }^{1}$, which include anticancer ${ }^{2}$, anti-inflammatory ${ }^{3}$, anticonvulsant ${ }^{4}$, and antidiuretic ${ }^{5}$ activities. Also quinazolinone such as tryptanthrin have been found to have remarkable antimalarial activity ${ }^{6}$.

1,2,4-Triazoles are associated with diverse pharmacological activities such as analgesic, antiasthmatic, diuretic, antihypersensitive, anticholinergic, antibacterial, antifungal and antiinflammatory activity $^{7-10}$. These biological data prompted us to synthesize some new quinazol-4-one derivatives containing 1,2,4- triazole ring.

\section{Experimental}

\section{General procedures}

Melting points were taken in open capillaries and are uncorrected. IR spectra $\left(\mathrm{KBr}\right.$ in $\left.\mathrm{cm}^{-1}\right)$ were recorded on Jasco 410 plus FTIR spectrophotometer. ${ }^{1} H$ NMR spectra were recorded on a JEOL $300 \mathrm{MHz}$ FT-NMR spectrophotometer using DMSO- $\mathrm{d}_{6}$ as solvent and TMS as 
internal standard (chemical shifts in $\delta \mathrm{ppm}$ ). The mass spectra of compounds were determined with Schimadzu model No.QP 2010. The elemental analysis was carried out on a Perkin Elmer $\mathrm{C}, \mathrm{H}, \mathrm{N}$ analyzer and sulphur analysis was obtained by oxygen-flask method. The purity of the compounds was monitored by thin layer chromatography. TLC was carried out on precoated $0.2 \mathrm{~mm}$ silica gel ${ }_{60} \mathrm{~F}^{254}$ plates.

\section{(4-Acetylamino-phenoxy)-acetic acid ethyl ester (2)}

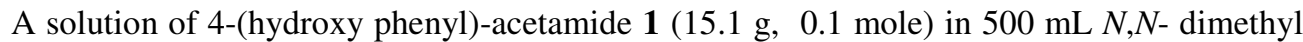
formamide was heated at $80-85{ }^{\circ} \mathrm{C}$ with ethyl chloroacetate $(13.47 \mathrm{~mL}, 0.11$ mole) in an oil bath in presence of anhydrous potassium carbonate $(13.8 \mathrm{~g}, 0.11$ mole). The reaction mixture was cooled and $1500 \mathrm{~mL}$ of cold water was added. A white-coloured crystalline solid separated was filtered, washed thoroughly first with cold water and then with cold ethanol, and finally recrystallised from $99 \%$ ethanol, yield $70 \%$, m.p. $104{ }^{\circ} \mathrm{C}$; IR ( $\mathrm{KBr}$ ) $3347(\mathrm{NH}), 1750(\mathrm{C}=\mathrm{O}$ ester $), 1589,1508,1477(\mathrm{C}=\mathrm{C}$, aromatic $)$.

\section{(4-Amino-phenoxy)-acetic acid (3)}

Compound 2 ( $23.7 \mathrm{~g}, 0.1$ mole) was heated with $35 \mathrm{~mL}$ concentrated hydrochloric acid and $100 \mathrm{~mL}$ water for about 2 hours. A clear solution obtained was filtered and neutralized with $40 \%$ of sodium acetate solution. The white crystalline solid precipitated out was filtered, washed thoroughly with water and recrystallised from DMF: ethanol (1:1), yield $72 \%$, m.p. $248{ }^{\circ} \mathrm{C}$; IR $(\mathrm{KBr}) 3464\left(\mathrm{NH}_{2}\right), 1650(\mathrm{C}=\mathrm{O}), 1558,1508,1445(\mathrm{C}=\mathrm{C}$, aromatic $)$.

\section{(4-Amino-phenoxy)-acetic acid ethyl ester (4)}

Compound 3 (11.85 g, 0.05 mole) was suspended in $250 \mathrm{~mL}$ ethanol and cooled to $10^{\circ} \mathrm{C}$. To this solution, sulphuric acid $(3.0 \mathrm{~mL}, 0.055 \mathrm{~mole})$ was added dropwise with stirring, maintaining the temperature at $10-15{ }^{\circ} \mathrm{C}$. The reaction mixture was then refluxed for about 2 hours. The excess ethanol was distilled under reduced pressure and then to one-third of the total volume of the solution, $100 \mathrm{~mL}$ water was added followed by $20 \%$ of potassium carbonate solution for neutralization. The product was extracted with $3 \times 25 \mathrm{~mL}$ of dichloromethane. The dichloromethane layer was washed with $3 \times 20 \mathrm{~mL}$ of water. The compound 4 obtained on distillation of dichloromethane at atmospheric pressure was dried in an oven at $40{ }^{\circ} \mathrm{C}$ and recrystallised from benzene, yield $80 \%$, m.p. $58{ }^{\circ} \mathrm{C}$; IR (KBr) 3382 $\left(\mathrm{NH}_{2}\right), 1740(\mathrm{C}=\mathrm{O}$ ester $), 1558,1509,1476(\mathrm{C}=\mathrm{C}$, aromatic $)$.

\section{[4-(4-Oxo-2-phenyl-4H-quinazolin-3-yl)-phenoxy]-acetic acid ethyl ester (5)}

A mixture of 2-phenyl-4H-3,1-benzoxazin-4-one (4.46 g, 0.02 mole) and (4-amino-phenoxy) acetic acid ethyl ester 4 (4.29 g, 0.022 mole) in $100 \mathrm{~mL}$ pyridine was refluxed for about 12 hours. The reaction mixture was then allowed to cool to room temperature and then added slowly $100 \mathrm{~mL}$ of methanol. The product 5 separated was filtered, washed with ethanol and crystallized from ethanol, yield $82 \%$, m.p. $180^{\circ} \mathrm{C}$; IR (KBr) $1735(\mathrm{C}=\mathrm{O}$ ester), $1650(\mathrm{CO} N)$, $1606(\mathrm{C}=\mathrm{N}), 1577,1544,1508,1448\left(\mathrm{C}=\mathrm{C}\right.$, aromatic); ${ }^{1} \mathrm{H}$ NMR (DMSO-d $\left.{ }_{6}\right) \delta 1.24(\mathrm{t}, 3 \mathrm{H}$, $\left.\mathrm{CH}_{3}\right), 4.1\left(\mathrm{q}, 2 \mathrm{H}, \mathrm{CH}_{2}\right), 4.7\left(\mathrm{~s}, 2 \mathrm{H}, \mathrm{O}^{\circ} \mathrm{CH}_{2} \mathrm{C}\right), 6.9-8.6(\mathrm{~m}, 13 \mathrm{H}, \mathrm{ArH})$; Anal.Calcd. for $\mathrm{C}_{24} \mathrm{H}_{20} \mathrm{~N}_{2} \mathrm{O}_{4}$ (400): C, 72.00; H, 5.00; N, 7.00. Found: C, 71.96; H, 5.02; N, 6.94.

\section{[4-(4-Oxo-2-phenyl-4H-quinazolin-3-yl)-phenoxy]-acetic acid hydrazide (6)}

Compound 5 ( $4.0 \mathrm{~g}, 0.01 \mathrm{~mole})$ and $2 \mathrm{~mL}$ of $99 \%$ hydrazine hydrate in ethanol was refluxed for about 8 hours. The reaction mixture was then allowed to cool to room temperature. The separated white coloured solid was filtered, washed with ethanol and crystallized from 
ethanol, yield $80 \%$, m.p. $220^{\circ} \mathrm{C}$; IR (KBr) 3315, $3272\left(\mathrm{NH}^{*} \mathrm{NH}_{2}\right), 1674$ (C=O amide), 1652 $\left(\mathrm{CO}{ }^{\circ}\right), 1603(\mathrm{C}=\mathrm{N}), 1585,1508,1450\left(\mathrm{C}=\mathrm{C}\right.$, aromatic); ${ }^{1} \mathrm{H}$ NMR (DMSO-d $\left.{ }_{6}\right) \delta 4.3(\mathrm{~s}$, $\left.2 \mathrm{H}, \mathrm{NH}_{2}\right), 4.4\left(\mathrm{~s}, 2 \mathrm{H}, \mathrm{O}^{\circ} \mathrm{CH}_{2} \cdot \mathrm{C}\right), 6.9-8.6(\mathrm{~m}, 13 \mathrm{H}, \mathrm{ArH}), 9.3(\mathrm{~s}, 1 \mathrm{H}, \mathrm{NH})$; Anal.Calcd. for $\mathrm{C}_{22} \mathrm{H}_{18} \mathrm{~N}_{4} \mathrm{O}_{3}$ (386): C, 68.39; H, 4.66; N, 14.50. Found: C, 68.41; H, 4.65; N, 14.52.

\section{[4-(4-Oxo-2-phenyl-4 $\underline{H}$-quinazolin-3-yl)-phenoxy]-acetic acid-N'-(substituted phenyl)-thiosemicarbazides (7a-d)}

Compound 6 (0.386g. 0.001 mole) was refluxed with various aromatic phenyl isothiocyanates $(0.0011$ mole) in isopropyl alcohol $(40 \mathrm{~mL})$ with continuous stirring over a period of 16 hours. The crystalline solid obtained on cooling the reaction mixture was filtered, washed with isopropyl alcohol and finally crystallized from ethanol to afford compounds (7a-d).

[4-(4-Oxo-2-phenyl-4H-quinazolin-3-yl)- phenoxy]-acetic acid- $N$ '-phenyl thiosemicarbazide (7a)

Colorless solid, yield $84 \%$, m.p. $195^{\circ} \mathrm{C}$; IR (KBr) $3230(\mathrm{NH}), 1678(\mathrm{CO} \times), 1662$ $\left(\mathrm{CH}_{2}{ }^{\circ} \mathrm{CO}\right), 1600(\mathrm{C}=\mathrm{N}), 1558,1508,1496,1446\left(\mathrm{C}=\mathrm{C}\right.$, aromatic); ${ }^{1} \mathrm{H}$ NMR (DMSO-d $\left.{ }_{6}\right) \delta$ $4.6\left(\mathrm{~s}, 2 \mathrm{H}, \mathrm{O}^{\circ} \mathrm{CH}_{2}{ }^{\circ} \mathrm{C}\right), 6.9-8.6(\mathrm{~m}, 18 \mathrm{H}, \mathrm{ArH}), 9.6(\mathrm{~s}, 1 \mathrm{H}, \mathrm{NH}), 9.7(\mathrm{~s}, 1 \mathrm{H}, \mathrm{NH}), 10.3(\mathrm{~s}, 1 \mathrm{H}$, $\mathrm{NH}$ ); Anal.Calcd. for $\mathrm{C}_{29} \mathrm{H}_{23} \mathrm{~N}_{5} \mathrm{O}_{3} \mathrm{~S}$ (521): C, 66.79; H, 4.41; N, 13.43; S, 6.14. Found: C, 66.74; H, 4.38; N, 13.52; S, 6.10.

[4-(4-Oxo-2-phenyl-4ㅁ-quinazolin-3-yl)-phenoxy]-acetic acid-N'-(4-fluoro-phenyl)thiosemicarbazide (7b)

Colorless solid, yield 86\%, m.p. $200^{\circ} \mathrm{C}$; IR (KBr) $3213(\mathrm{NH}), \quad 1697\left(\mathrm{CH}_{2}{ }^{\circ} \mathrm{CO}\right), 1683$ $(\mathrm{CO} N), 1602(\mathrm{C}=\mathrm{N}), 1585,1508,1489\left(\mathrm{C}=\mathrm{C}\right.$, aromatic); ${ }^{1} \mathrm{H}$ NMR $\left(\mathrm{DMSO}_{6} \mathrm{~d}_{6}\right) \delta 4.6(\mathrm{~s}$, $\left.2 \mathrm{H}, \mathrm{O}^{\circ} \mathrm{CH}_{2}{ }^{\circ} \mathrm{C}\right), 6.9-8.6(\mathrm{~m}, 17 \mathrm{H}, \mathrm{ArH}), 9.6(\mathrm{~s}, 1 \mathrm{H}, \mathrm{NH}), 9.7(\mathrm{~s}, 1 \mathrm{H}, \mathrm{NH}), 10.3(\mathrm{~s}, 1 \mathrm{H}, \mathrm{NH})$; Anal.Calcd. for $\mathrm{C}_{29} \mathrm{H}_{22} \mathrm{FN}_{5} \mathrm{O}_{3} \mathrm{~S}$ (539): C, 64.56; H, 4.08; N, 12.77; S, 5.93. Found: C, 64.59; $\mathrm{H}, 4.11 ; \mathrm{N}, 12.82 ; \mathrm{S}, 6.00$.

\section{[4-(4-Oxo-2-phenyl-4ㅂ-quinazolin-3-yl)-phenoxy]-acetic acid-N'-(4-nitro-phenyl)-} thiosemicarbazide $(7 \mathrm{c})$

Pale yellow coloured solid, yield $80 \%$, m.p. $180^{\circ} \mathrm{C}$; IR (KBr) $3286(\mathrm{NH}), 1701\left(\mathrm{CH}_{2} \mathrm{CO}\right)$, $1647(\mathrm{CO} \times), 1597(\mathrm{C}=\mathrm{N}), 1587,1556,1508,1496\left(\mathrm{C}=\mathrm{C}\right.$, aromatic); ${ }^{1} \mathrm{H}$ NMR (DMSO-d $\left.{ }_{6}\right)$ $\delta 4.6\left(\mathrm{~s}, 2 \mathrm{H}, \mathrm{OCH}_{2} \cdot \mathrm{C}\right), 6.9-8.6(\mathrm{~m}, 17 \mathrm{H}, \mathrm{ArH}), 10.0(\mathrm{~s}, 1 \mathrm{H}, \mathrm{NH}), 10.1(\mathrm{~s}, 1 \mathrm{H}, \mathrm{NH}), 10.4(\mathrm{~s}$, $1 \mathrm{H}, \mathrm{NH}$ ); Anal.Calcd. for $\mathrm{C}_{29} \mathrm{H}_{22} \mathrm{~N}_{6} \mathrm{O}_{5} \mathrm{~S}$ (566): C, 61.48; H, 3.88; N, 14.84; S, 5.65. Found: C, 61.45; H, 4.39; N, 14.82; S, 5.70.

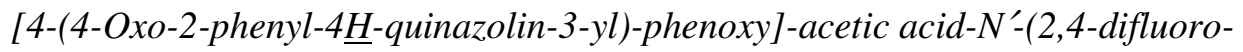
phenyl)-thiosemicarbazide (7d)

Colorless solid, yield $78 \%$, m.p. 202 ${ }^{\circ} \mathrm{C}$; IR (KBr) $3250(\mathrm{NH}), 1662\left(\mathrm{CH}_{2} \mathrm{CO}\right), 1635(\mathrm{CO} N), 1600$ $(\mathrm{C}=\mathrm{N}), 1583,1558,1508,1485\left(\mathrm{C}=\mathrm{C}\right.$, aromatic); ${ }^{1} \mathrm{H}$ NMR (DMSO-d $\left.{ }_{6}\right) \delta 4.6\left(\mathrm{~s}, 2 \mathrm{H}, \mathrm{O}^{\circ} \mathrm{CH}_{2} \mathrm{C}\right), 6.9-8.6$ (m, 16H, ArH), 9.7 (s, $1 \mathrm{H}, \mathrm{NH}), 9.8$ (s, $1 \mathrm{H}, \mathrm{NH}), 10.3$ (s, $1 \mathrm{H}, \mathrm{NH}$ ); Anal.Calcd. for $\mathrm{C}_{29} \mathrm{H}_{21} \mathrm{~F}_{2} \mathrm{~N}_{5} \mathrm{O}_{3} \mathrm{~S}$ (557): C, 62.47; H, 3.77; N, 12.56; S, 5.74. Found : C, 62.51; H, 3.76; N, 12.62; S, 5.78.

\section{3-[4-(4-Substituted phenyl-5-thioxo-4,5-dihydro-1ㅂ-[1,2,4]triazol-3-yl-methoxy)- phenyl]-2-phenyl-3ㅂ-quinazolin-4-ones $(\mathbf{8 a - d})$}

To the hot reaction mixture of compounds (7a-d, 0.0005 mole) in $50 \mathrm{~mL}$ of ethanol was added sodium metal $(0.001$ mole) pieces and the reaction mixture was refluxed with continuous stirring over a period of 4 hours. The excess ethanol was distilled under 
atmospheric pressure till 3-4 $\mathrm{mL}$ was left behind. The viscous liquid obtained on cooling was poured into $20 \mathrm{~mL}$ of cold water, followed by neutralized with 1:1 hydrochloric acid under cold condition. The product obtained was filtered, washed thoroughly with water and crystallised from ethanol to give compounds (8a-d).

3-[4-(4-Phenyl-5-thioxo-4,5-dihydro-1ㅁ-[1,2,4]triazol-3-yl-methoxy)-phenyl]-2phenyl-3ㅍ-quinazolin-4-one (8a)

Colorless solid ,yield $56 \%$, m.p. $265^{\circ} \mathrm{C}$; IR (KBr) 3429 (NH), 1667 (CO'N), $1602(\mathrm{C}=\mathrm{N})$, $1585,1508,1450\left(\mathrm{C}=\mathrm{C}\right.$, aromatic); ${ }^{1} \mathrm{H}$ NMR $\left(\mathrm{DMSO}_{6} \mathrm{~d}_{6}\right) \delta 5.0\left(\mathrm{~s}, 2 \mathrm{H}, \mathrm{O}^{\circ} \mathrm{CH}_{2}{ }^{\circ} \mathrm{C}\right), 6.9-8.6$ (m, 18H, ArH), $14.0(\mathrm{~s}, 1 \mathrm{H}, \mathrm{NH})$; MS (m/z): $503\left[\mathrm{M}^{+}\right], 371,327,313,223,205,179,166$, 146, 118, 109, 105, 90, 77; Anal.Calcd. for $\mathrm{C}_{29} \mathrm{H}_{21} \mathrm{~N}_{5} \mathrm{O}_{2} \mathrm{~S}$ (503): C, 69.18; H, 4.17; N, 13.91; S, 6.36. Found: C, 69.12; H, 4.19; N, 13.98; S, 6.40.

\section{3-\{4-[4-(4-Fluoro-phenyl)-5-thioxo-4,5-dihydro-1ㅂ-[1,2,4]triazol-3-yl-methoxy]-} phenyl\}-2-phenyl-3ㅍ-quinazolin-4-one $(8 \boldsymbol{b})$

Colorless solid, yield $65 \%$, m.p. $298^{\circ} \mathrm{C}$; IR (KBr) $3326(\mathrm{NH}), 1637\left(\mathrm{CO}{ }^{\circ}\right), 1603(\mathrm{C}=\mathrm{N})$, $1587,1558,1508,1450\left(\mathrm{C}=\mathrm{C}\right.$, aromatic); ${ }^{1} \mathrm{H}$ NMR $\left(\mathrm{DMSO}^{-} \mathrm{d}_{6}\right) \delta 5.0\left(\mathrm{~s}, 2 \mathrm{H}, \mathrm{OCCH}_{2}{ }^{\circ} \mathrm{C}\right)$, 6.9-8.6 (m, 17H, ArH), 14.0 (s, 1H, NH); MS (m/z): $521\left[\mathrm{M}^{+}\right], 371,327,313,223,205,179$, 166, 146, 118, 109, 105, 90, 77; Anal.Calcd. for $\mathrm{C}_{29} \mathrm{H}_{20} \mathrm{FN}_{5} \mathrm{O}_{2} \mathrm{~S}$ (521): C, 66.79; H, 3.83; N, 13.43; S, 6.14. Found: C, 66.75; H, 3.80; N, 13.49; S, 6.21.

\section{3-\{4-[4-(4-Nitro-phenyl)-5-thioxo-4,5-dihydro-1ㅂ-11,2,4]triazol-3-yl-methoxy]- phenyl\}-2-phenyl-3ㅍ-quinazolin-4-one (8c)}

Pale yellow coloured solid, yield $59 \%$, m.p. $185^{\circ} \mathrm{C}$; IR (KBr) $3314(\mathrm{NH}), 1648\left(\mathrm{CO}{ }^{*} \mathrm{~N}\right)$, $1600(\mathrm{C}=\mathrm{N}), 1587,1508,1449\left(\mathrm{C}=\mathrm{C}\right.$, aromatic); ${ }^{1} \mathrm{H}$ NMR $\left(\mathrm{DMSO}_{6}\right) \delta 4.7(\mathrm{~s}, 2 \mathrm{H}$, $\left.\mathrm{O}^{\cdot} \mathrm{CH}_{2}{ }^{\circ} \mathrm{C}\right), 6.9-8.6(\mathrm{~m}, 17 \mathrm{H}, \mathrm{ArH}), 14.0(\mathrm{~s}, 1 \mathrm{H}, \mathrm{NH}) ; \mathrm{MS}(\mathrm{m} / \mathrm{z}): 548\left[\mathrm{M}^{+}\right], 371,327,313$, 223, 205, 179, 166, 146, 118, 109, 105, 90, 77; Anal.Calcd. for $\mathrm{C}_{29} \mathrm{H}_{20} \mathrm{~N}_{6} \mathrm{O} \mathrm{O}_{4} \mathrm{~S}$ (548): $\mathrm{C}$, 63.50; H, 3.64; N, 15.32; S, 5.83. Found: C, 63.52; H, 3.66; N, 15.39; S, 5.80.

3-\{4-[4-(2,4-Difluoro-phenyl)-5-thioxo-4,5-dihydro-1H-[1,2,4]triazol-3-yl-ethoxy]phenyl\}-2-phenyl-3ㅂ-quinazolin-4-one $(8 \mathrm{~d})$

Colorless solid, yield $61 \%$, m.p. $240^{\circ} \mathrm{C}$; IR (KBr) $3332(\mathrm{NH}), 1647(\mathrm{CO} \cdot \mathrm{N}), 1602(\mathrm{C}=\mathrm{N})$, 1587, 1509, $1449\left(\mathrm{C}=\mathrm{C}\right.$, aromatic); ${ }^{1} \mathrm{H}$ NMR $\left(\mathrm{DMSO}_{6} \mathrm{~d}_{6}\right) \delta 5.0\left(\mathrm{~s}, 2 \mathrm{H}, \mathrm{O}^{\circ} \mathrm{CH}_{2}{ }^{\circ} \mathrm{C}\right), 6.9-8.6$ $(\mathrm{m}, 16 \mathrm{H}, \mathrm{ArH}), 14.0(\mathrm{~s}, 1 \mathrm{H}, \mathrm{NH}) ; \mathrm{MS}(\mathrm{m} / \mathrm{z}): 539\left[\mathrm{M}^{+}\right], 371,327,313,223,205,179,166$, 146, 118, 109, 105, 90, 77; Anal.Calcd. for $\mathrm{C}_{29} \mathrm{H}_{19} \mathrm{~F}_{2} \mathrm{~N}_{5} \mathrm{O}_{2} \mathrm{~S}$ (539): C, 64.56; H, 3.52; N, 12.98; S, 5.93. Found: C, 64.51; H, 3.49; N, 13.05; S, 5.90.

\section{Results and Discussion}

4-Hydroxy-phenyl acetamide $\mathbf{1}$ on electrophilic substitution with ethyl chloroacetate gave (4-acetylamino-phenoxy)-acetic acid ethyl ester $\mathbf{2}$ which on hydrolysis with aqueous hydrochloric acid gave 4-(amino-phenoxy)-acetic acid 3. This on further esterification with sulphuric acid gave 4-(amino-phenoxy)-acetic acid ethyl ester ${ }^{11} 4$.

The 2-phenyl-4ㅂ-3,1-benzoxazin-4-one ${ }^{12,13}$ which was prepared from 2-amino anthranilic acid and benzoyl chloride at $20-25{ }^{\circ} \mathrm{C}$ in pyridine was allowed to react with 4 (amino-phenoxy)-acetic acid ethyl ester 4 to give [4-(4-oxo-2-phenyl-4H-quinazolin-3-yl)phenoxy]-acetic acid ethyl ester 5. The structure of compound $\mathbf{5}$ was established by analytical and spectral data. The compound $\mathbf{5}$ was then condensed with hydrazine hydrate in ethanol to afford [4-(4-oxo-2-phenyl-4H-quinazolin-3-yl)-phenoxy]-acetic acid hydrazide 6 
in good yields. The compound $\mathbf{6}$ on reaction with various phenyl isothiocyanates in isopropyl alcohol gave [4 -(4-oxo-2-phenyl-4ㅂ-Huinazolin-3-yl)-phenoxy]- acetic acid- $\mathrm{N}^{\prime}$ - (substituted phenyl)-thiosemicarbazides (7a-d). The presence of NH group is confirmed by IR spectra which showed characteristic band at $3300 \mathrm{~cm}^{-1}$ and ${ }^{1} \mathrm{H}$ NMR signal at $\delta 9.6,9.7$ and 10.3 corresponding to $\mathrm{O}=\mathrm{C} \cdot \mathrm{NH} \cdot \mathrm{NH} \cdot \mathrm{C}=\mathrm{S} \cdot \mathrm{NH}$ protons. The compounds (7a-d) on Cyclisation with sodium metal in ethanol gave 3-[4-(4-substituted phenyl-5-thioxo-4,5-dihydro-1ㅍ-[1,2,4]-triazol- 3-yl- methoxy)phenyl]-2-phenyl-3ㅍ-quinazolin-4-ones (8a-d) [Scheme 1]. IR spectra of compounds (8a-d) showed the presence of $\mathrm{NH}$ group between at $3300 \mathrm{~cm}^{-1}$ and $3100 \mathrm{~cm}^{-1}$ and also the ${ }^{1} \mathrm{H}$ NMR signal at $\delta 14.0$ indicates the presence of $\mathrm{NH}$ group for structures of compounds $(\mathbf{8 a - d})$.
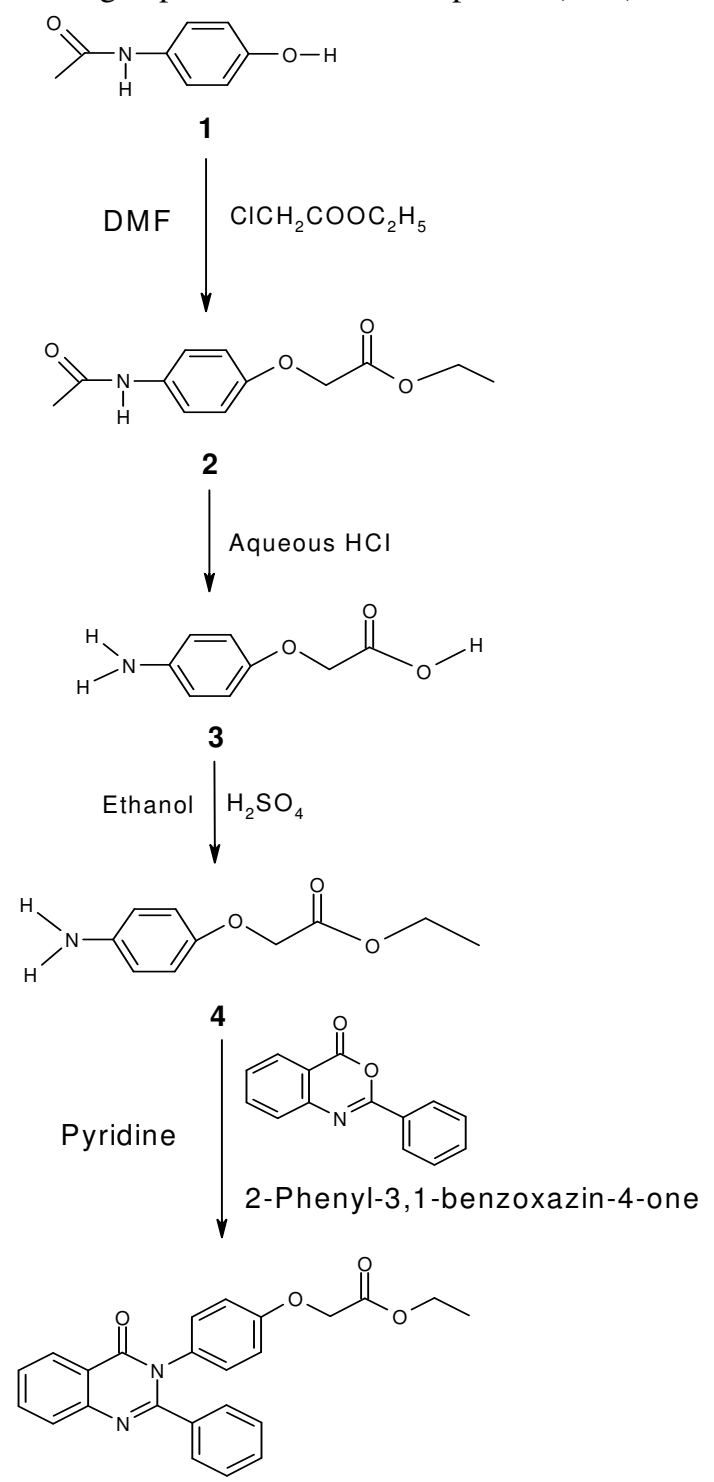

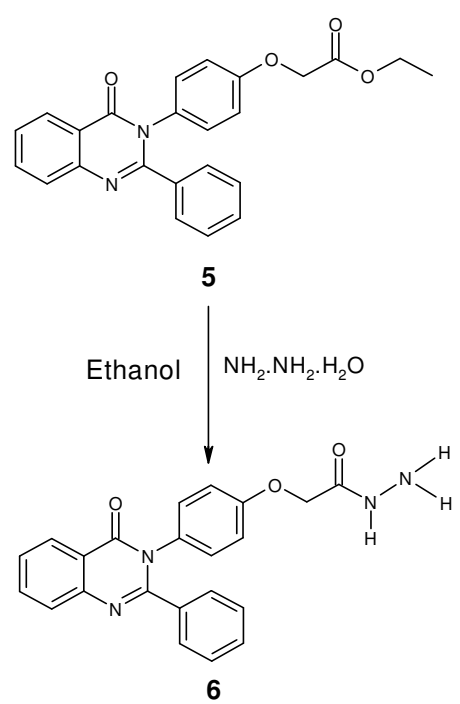

$\begin{array}{ll}\text { 7a } & \mathrm{R} 1=\mathrm{R} 2=\mathrm{H} \\ \text { 7b } & \mathrm{R} 1=\mathrm{H}, \mathrm{R} 2=\mathrm{F} \\ \text { 7c } & \mathrm{R} 1=\mathrm{H}, \mathrm{R} 2=\mathrm{NO}_{2} \\ \text { 7d } & \mathrm{R} 1=\mathrm{F}, \mathrm{R} 2=\mathrm{F}\end{array}$
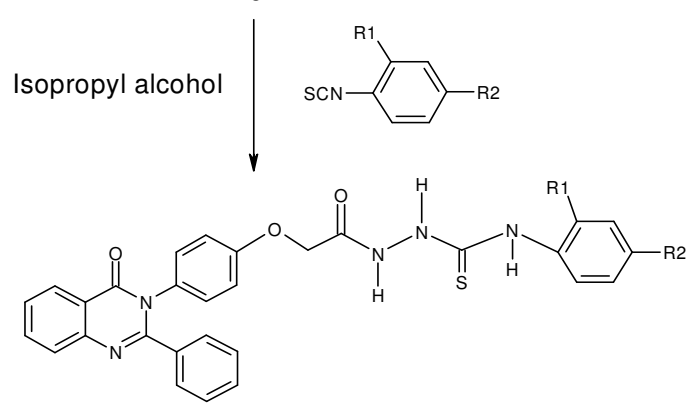

(7a-d)<smiles></smiles>

$(8 a-d)$

Scheme 1

\section{Biological Activity}

$$
\begin{array}{ll}
\text { 8a } & R 1=R 2=H \\
\text { 8b } & R 1=H, R 2=F \\
\text { 8c } & R 1=H, R 2=\mathrm{NO}_{2} \\
\text { 8d } & R 1=F, R 2=F
\end{array}
$$

\section{Antibacterial activity}

All the newly synthesized compounds (8a-d) were screened in vitro for their antibacterial activity against Staphylococcus aureus, Escherichia coli and Bacillus subtilis by the ditchplate technique ${ }^{20}$ using concentrations of $50 \mu \mathrm{g} / \mathrm{mL}$. Nutrient agar was employed as culture media and DMF was used as solvent control for antibacterial activity. 


\section{Antifungal activity}

The compounds (8a-d) synthesized were screened for their antifungal activity against Aspergillus niger, Candida albicans and Cryptococcus neoformans by paper-disc diffusion method $^{21}$ at concentrations of $50 \mu \mathrm{g} / \mathrm{mL}$. Nutrient agar was employed as culture media and DMF was used as solvent control for antifungal activity.

The known compounds, such as ampicillin, amoxicillin, norfloxacin, penicillin and Griseofulvin were used for comparison purpose. The diameter of zone of inhibition was measured in $\mathrm{mm}$. The antibacterial and antifungal screening data are recorded in Table 1.

Table 1. Biological activity data

\begin{tabular}{|c|c|c|c|c|c|c|}
\hline \multirow{3}{*}{ Compounds } & \multicolumn{6}{|c|}{ Zone of nhibition inmm } \\
\hline & \multicolumn{3}{|c|}{ Antibacterial activity } & \multicolumn{3}{|c|}{ Antifungal activity } \\
\hline & S.aureus & E.coli & B. subtilis & A. niger & C. albicans & C.neoformans \\
\hline $8 a$ & 11 & 10 & 11 & 12 & 14 & 16 \\
\hline $8 b$ & 13 & 14 & 16 & 14 & 11 & 15 \\
\hline $8 \mathrm{c}$ & 17 & 12 & 18 & 16 & 14 & 18 \\
\hline $8 \mathrm{~d}$ & 16 & 15 & 13 & 11 & 15 & 12 \\
\hline
\end{tabular}

From the Table 1, it can be seen that the compounds $\mathbf{8 c}$ and $\mathbf{8 d}$ showed remarkable activity against Staphylococcus aureus, compounds $\mathbf{8 b}$ and $\mathbf{8 c}$ showed remarkable activity against Bacillus subtilis. The compounds $\mathbf{8 c}$ showed good activity against A.niger and the compounds $\mathbf{8 a}$ and $\mathbf{8 c}$ showed maximum activity against cryptococcus neoformans.

\section{Antimalarial activity}

The effort to find new antimalarial activity is still a high priority given to the increasing malarial emergency due to chloroquine resistant Plasmodium falciparum strains. The chloroquine-resistant Plasmodium falciparum malarial parasite was cultured in vitro and the sensitivity of parasite to the newly synthesized compounds was evaluated using the tritiated Hypoxanthine incorporation assay ${ }^{16}$. The compounds (8a-d) were tested for antimalarial activity and the only compound 3-\{4-[4-(4-fluoro-phenyl)- $4 \underline{H}-[1,2,4]$ triazol-3-yl-methoxy]phenyl $\}$-2-phenyl-3 $\underline{H}$-quinazolin-4-one $8 \mathrm{~b}$ was found to be most active against Plasmodium falciparum strains and its $50 \%$ inhibitor concentration (IC50) values were $1.2 \mu \mathrm{M}$.

\section{Acknowledgement}

The authors are thankful to TIFR and Institute of Science, Mumbai for ${ }^{1} \mathrm{H}$ NMR and mass spectra and Dr. (Mrs.) Vivien Amonkar, Head, Department of Microbiology, St. Xavier's College, Mumbai for providing biological activity.

\section{References}

1. (a) Wolfe J F, Rathman T L, Sleevi MC, Campbell J A and Greenwood T D, J. Med. Chem. 1990, 33, 161. (b) Padia J K, Field M, Hilton J, Meecham K, Pablo J, Pinnock R, Roth B D, Singh L, Suman-Chauhan N, Trivedi B K and Webdale L, J. Med. Chem. 1998, 41, 1042.

2. Xia Y, Yang Z Y, Hour M J, Kuo S C, Xia P, Bastow K F, Nakanishi Y, Nampoothiri P, Hackl T, Hamel E and Lee K H, Bioorg. Med. Chem. Lett. 2001, 11, 1193.

3. Kenichi O, Yoshihisa Y, Toyonari O, Toru I and Yoshio I, J. Med. Chem. 1985, 28, 568.

4. Buchanan J G, Sable H Z and Thygarajan B S Ed.; Wiley-Interscience: New York, 1972, 2, pp.1-96. 
5. Lyle F R, US Pat. 5,973,257. Chem. Abstr. 1985, 65, 2.

6. Ridley R G, Sciences 1999, 285, 1502.

7. Birendra N G, Jiban C S K and Jogendra N B, J. Heterocycl. Chem. 1984, 21, 1225.

8. (a) Kothari P J, Mehlhoff M A, Singh S P, Parmar S S and Stenberg V I J.Heterocycl. Chem. 1980, 17, 1369. (b) Kothari P J, Singh S P, Parmar S S and Stenberg V I, J. Heterocycl. Chem. 1980, 17, 1393.

9. Sengupta A K and Misra H K, J. Indian Chem. Soc. 1981, 8, 508.

10. Sarmah S C and Bahel S C, J. Indian Chem. Soc. 1982, 59, 877.

11. Walter A, Heidelberger, Jacobs and Michael, J. American Chem. Soc. 1917, 39, 2196.

12. Tiwari S S and Satsangi R K, J. Indian Chem. Soc. 1975, 55, 736.

13. Zentmyer D T and Wagner E C, J. Org. Chem. 1949, 14, 967.

14. Collins $\mathrm{C} \mathrm{H}$ and Lyne P M, Microbiological Methods; $3^{\text {rd }}$ Ed; Butterworths, London, 1970, p. 424.

15. Seeley H W and Van Denmark P J, Microbes in Action, W.H. Freeman and Co. 1972, USA.

16. Desjardins R E, Canfield C J, Hayens J D and Chulay J D, Antimicrob Agents Chemother 1979, 16, 710. 


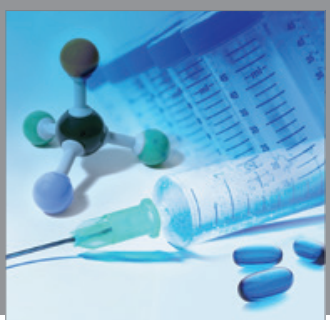

International Journal of

Medicinal Chemistry

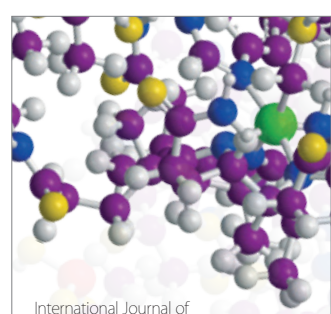

Carbohydrate Chemistry

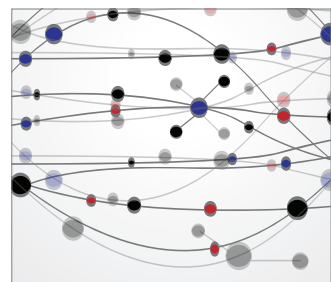

The Scientific World Journal
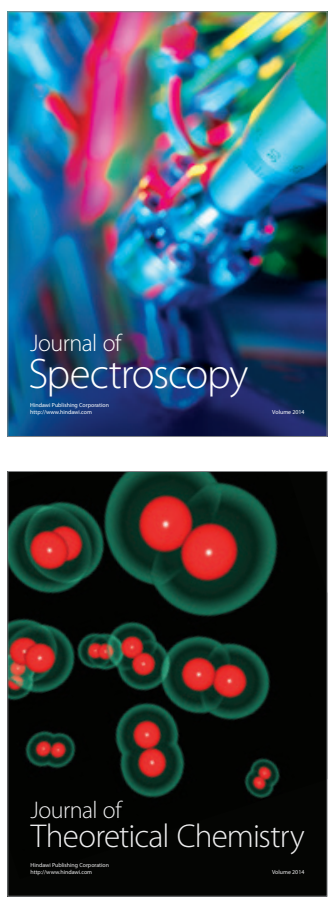
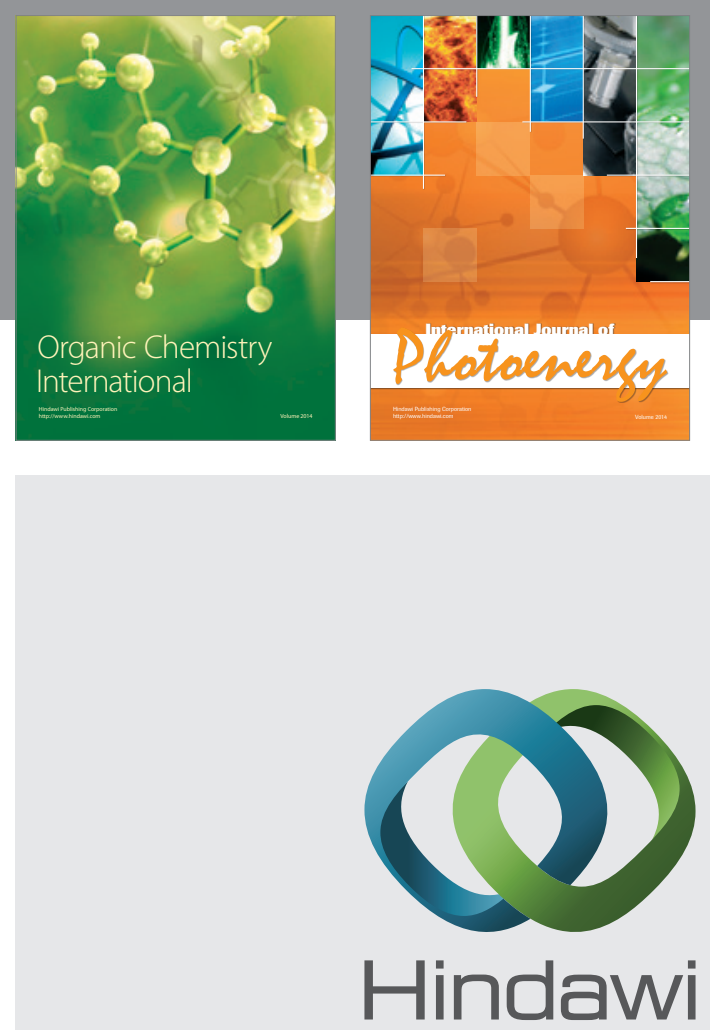

Submit your manuscripts at

http://www.hindawi.com
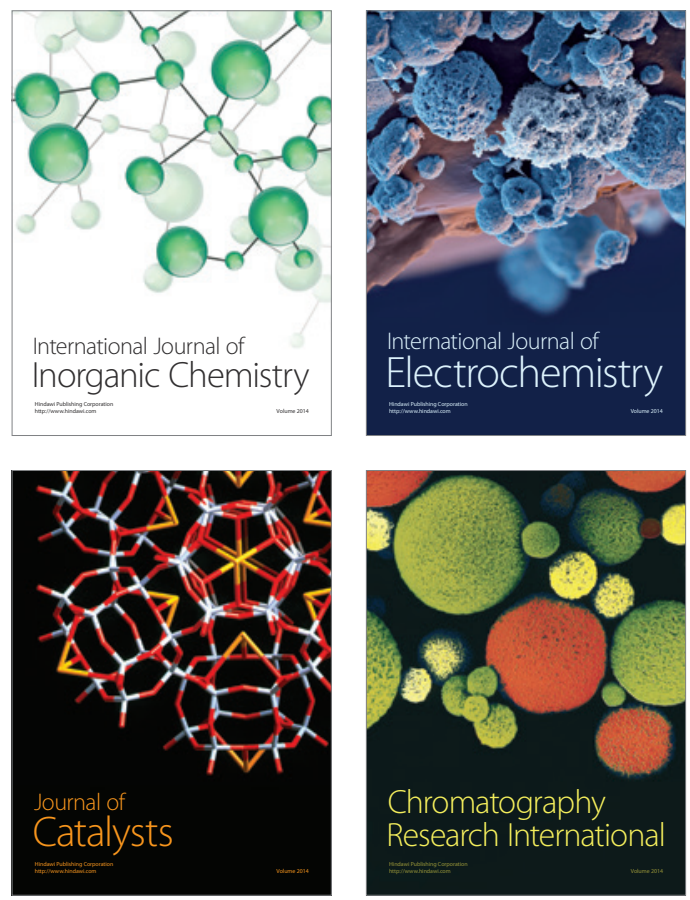
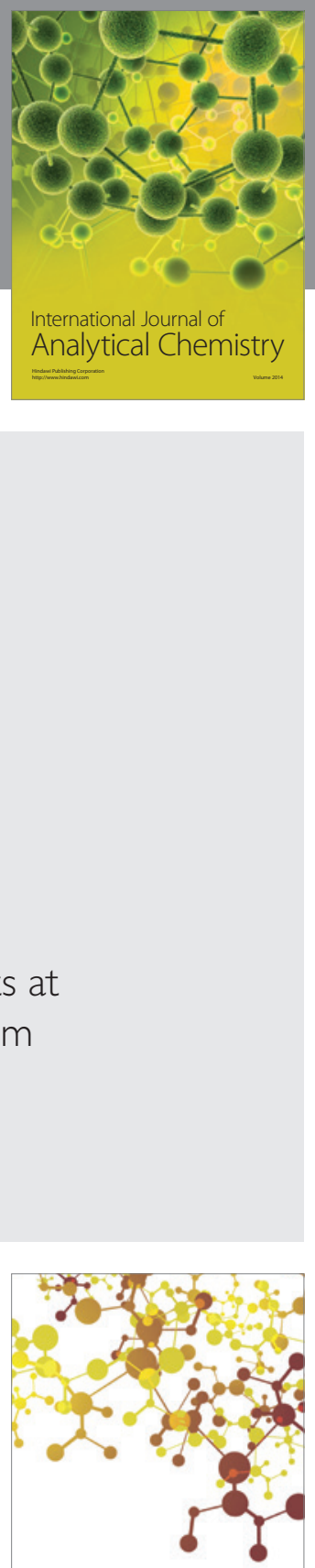

Journal of

Applied Chemistry
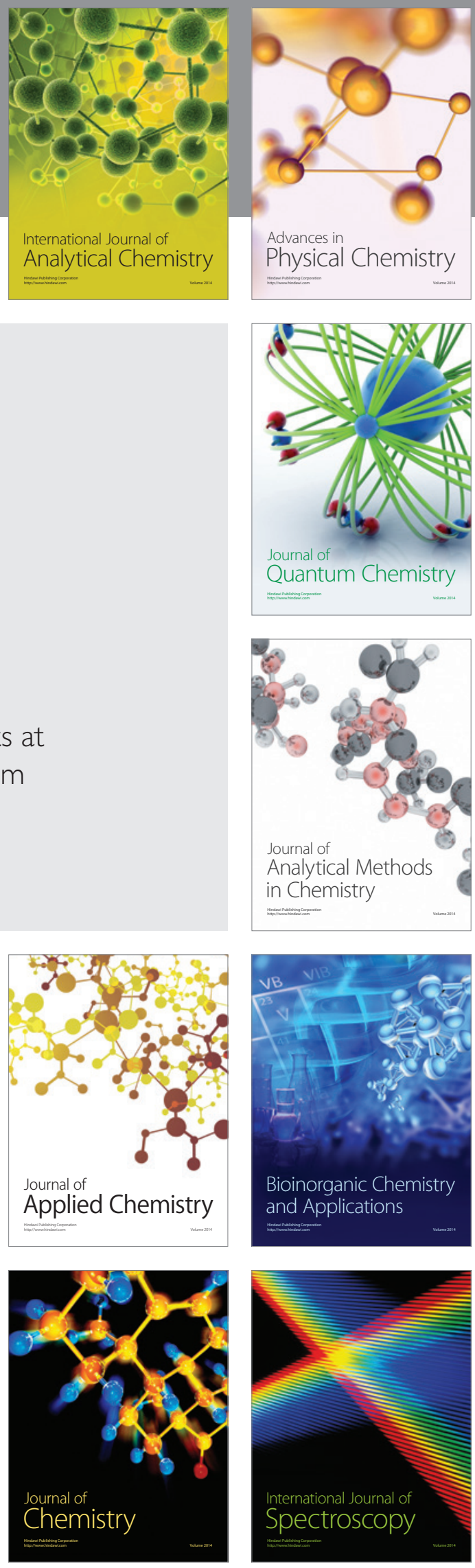